Digestive endoscopy

\title{
Oesophagogastroduodenoscopy in patients with cirrhosis: Extending the range of detection beyond portal hypertension
}

\author{
Stefania De Lisi*, Sergio Peralta, Andrea Arini, Fabio Simone, Antonio Craxì \\ Cattedra É Unità Operativa di Gastroenterologia, Di.Bi.M.I.S., University of Palermo, Palermo, Italy
}

\section{A R T I C L E I N F O}

\section{Article history:}

Received 8 February 2010

Accepted 2 April 2010

Available online 14 May 2010

\section{Keywords:}

Peptic ulcer

Screening oesophageal varices

\begin{abstract}
A B S T R A C T
Background: Oesophagogastroduodenoscopy is currently recommended for the screening of varices in cirrhosis. In addition to the assessment of varices, oesophagogastroduodenoscopy can detect conditions that, while unrelated to portal hypertension, may require treatment.

Aims: We evaluated in a large cohort of cirrhotic patients the prevalence of upper digestive findings other than oesophagogastric varices, the associations between upper gastrointestinal findings, portal hypertension and features of cirrhosis, and the incidence of new lesions in the course of a surveillance program.

Methods: Analysis of the records of 611 consecutive cirrhotic patients undergoing oesophagogastroduodenoscopy for screening and surveillance.

Results: 232 patients (38\%) presented endoscopic lesions not related to portal hypertension: peptic diseases $(n=193)$, proliferative diseases $(n=27)$ and vascular diseases $(n=12)$. In the screening group, 127 patients (39.4\%) had pathologic lesions. At multivariate analysis, an association was found between peptic diseases and the absence of portal hypertensive gastropathy (RR 3.3; 95\% CI 2.2-4.8); vascular diseases were associated with endoscopic signs of portal hypertension $(p=0.01)$. During surveillance, $9 / 55$ patients (16.3\%) in the group without previous pathologic findings developed new lesions.

Conclusions: Oesophagogastroduodenoscopy in patients with cirrhosis undergoing endoscopy for screening diagnosed pathologic lesions unrelated to portal hypertension requiring a change in management in $39.4 \%$ of asymptomatic subjects.
\end{abstract}

(C) 2010 Editrice Gastroenterologica Italiana S.r.l. Published by Elsevier Ltd. All rights reserved.

\section{Introduction}

Oesophagogastroduodenoscopy (EGD) is the gold standard for the diagnosis of oesophageal varices (EV), and is currently recommended by guidelines as a screening tool for portal hypertension once a diagnosis of cirrhosis is made. The size of varices and the presence of red wale marks on varices are used to predict the need for the prophylaxis of beta-blockers for bleeding. The presence and size of varices, and the stage of cirrhosis, dictate the intervals for repeating EGD [1-5].

Screening all patients with cirrhosis by endoscopy detects medium/large varices, i.e. those at significant risk of bleeding, in $15-25 \%$ of patients [6]. EGD implies an economic burden and has a degree of invasiveness. In recent years, the growing interest in non-endoscopic screening of portal hypertension has led to the development of several alternative models for predicting the presence of high risk varices in order to avoid EGD

\footnotetext{
* Corresponding author at: Gastroenterologia \& Epatologia, Piazza delle Cliniche 2, 90127 Palermo, Italy. Tel.: +39 091655 2280; fax: +39 0916552156.

E-mail address: stefaniadelisi@gmail.com (S. De Lisi).
}

in those patients who do not require primary prophylaxis for bleeding. Earlier studies evaluated the role of biochemical and sonographic parameters, such as platelet count/spleen diameter ratio [7], and Fibrotest [8]. More recently, transient elastography (Fibroscan), computerized tomography (CT) and video capsule endoscopy have been assessed. Fibroscan measurements establish a good correlation between liver stiffness and portal hypertension as measured by the hepatic venous pressure gradient (HVPG), but no correlation with variceal size $[9,10]$. Multidetector CT (MCT) oesophagography can detect the presence of EV and measure their size, with good agreement between radiologists and endoscopists, and generates better patient compliance than that found with unsedated EGD [11]. A further cost-effectiveness analysis of MCT oesophagography in comparison to EGD in cirrhotic patients concluded that MCT is more cost-effective than EGD for screening [12]. A recent multicenter trial that compared video capsule endoscopy to EGD suggested that the former has higher sensitivity and specificity in detecting varices [13], and it has been shown to be cost-effective compared to EGD [14]. Its availability is, however, currently limited in most clinical settings, and its inability to sample tissues may be a limitation in diagnosis. 
In the setting of cirrhosis, in addition to oesophageal and gastric varices, lesions of the upper digestive tract are frequently found. Some, such as portal hypertensive gastropathy (PHG), are common, and are clearly relevant to prognosis and treatment of the liver disease [15-17]. Others, such as peptic ulcers and gastric cancer, have a higher prevalence than that found in the general population $[18,19]$.

Should non-invasive diagnostic techniques became the screening tool for clinically significant portal hypertension in cirrhosis, the number of EGDs performed in these patients would dramatically decrease. It is therefore important to know the actual prevalence of pathologic findings of the upper digestive tract other than oesophagogastric varices, which would determine a change in management of cirrhotic patients. In this study, the aim was to evaluate: (a) the prevalence of non-variceal pathologic findings in a large cohort of cirrhotic patients undergoing EGD for screening and surveillance, (b) the possible associations among upper gastrointestinal findings, portal hypertension and liver disease characteristics, and (c) the incidence of new lesions not related to portal hypertension during the endoscopic surveillance program.

\section{Materials and methods}

Data from all consecutive patients with liver cirrhosis undergoing EGD at our tertiary Academic Liver Unit, from January 2006 to April 2009, were collected in an ad hoc database. The diagnosis of cirrhosis was based either on a combination of clinical, biochemical and imaging methods $(n=474)$ or on liver biopsy $(n=137)$.

The following epidemiological and clinical data were registered: age, gender, aetiology of cirrhosis, time of diagnosis, previous variceal bleeding and treatments for portal hypertension, previous endoscopic findings, gastrointestinal symptoms and current therapy, including proton pump inhibitors (PPI), nonsteroidal antiinflammatory drugs (NSAIDs), anticoagulants and beta-blockers. Biochemical data (total bilirubin, albumin, international normalized ratio (INR), haemoglobin and platelets) were also included. Spleen bipolar diameter was measured by standard B-mode ultrasonography.

An aetiology of cirrhosis was defined as viral (HBsAg positive and/or anti-HCV positive), alcoholic (current or historical ethanol intake of over $60 \mathrm{~g} /$ day), autoimmune according to defined criteria [20-22], post non-alcoholic fatty liver disease (NAFLD) if metabolic syndrome was present [23], and cryptogenic when no possible cause was identified. The severity of liver disease was classified according to the Child-Pugh score at the time of EGD [24].

All EGDs were performed by three endoscopists with experience in portal hypertension.

The timing for EGD was established according to the current guidelines $[2,4]$.

The presence and size of EV were graded as small, medium or large according to the system proposed by the North Italian Endoscopic Club for the Study and Treatment of Esophageal Varices [25]. Classification of gastric varices was based on their relationship with EV, as well as their location in the stomach [26]. The presence and severity of PHG were scored according to the New Italian Endoscopy Conference criteria as either mild or severe [27]. Presence and severity of oesophagitis were graded according to the Los Angeles classification [28]. Gastric and duodenal ulcers were diagnosed as the presence of a crater $>3 \mathrm{~mm}$; the presence of bleeding and the base characteristics were graded according to the Forrest classification [29]. Gastritis and duodenitis were defined as the presence of erythema, oedema and mucosal friability with or without mucosal erosions. Rapid urease test and/or histology from both antral and gastric body biopsies were performed in patients with endoscopic findings suggestive of Helicobacter pylori (H. pylori) infection. Gastric antral vascular ectasia (GAVE) was diagnosed by the presence of flat, or slightly raised, red stripe-like lesions radiating from the pylorus to the antrum [30]. Gastric angiodysplasia was not reported because of the high risk of confusion with mucosal changes occurring in PHG. Gastric and duodenal polyps were defined as circumscribed, pedunculated or sessile lesions $\geq 5 \mathrm{~mm}$.

Biopsies were performed when coagulation parameters were not severely impaired.

As recommendations for medium size EV are the same as those for large varices, we considered two categories of EV: small and medium/large. Upper digestive findings not related to portal hypertension were grouped according to their pathogenesis in: peptic diseases (oesophagitis, gastritis, duodenitis and peptic ulcers), proliferative diseases (gastroduodenal polyps and cancer), and vascular diseases (GAVE syndrome and duodenal angiodysplasias).

\subsection{Statistical analysis}

Data were obtained through retrospective review of the database and of clinical records. The demographics and clinical characteristics were analysed using the Student's $t$-test, analysis of the variance, and $\chi^{2}$ test, as appropriate. Multiple logistic regression analysis was used to identify predictive variables of upper digestive lesions not related to portal hypertension. The Kaplan-Meier method was applied to examine the incidence of new lesions over the course of the surveillance program.

All tests were two-sided, and a value of $p<0.05$ was considered statistically significant. Statistical calculations were made with the SPSS statistical package for Windows, version 13.0 (SPSS Inc., Chicago, IL, USA).

\section{Results}

611 patients underwent a single EGD between January 2006 and April 2009; 346 (56.6\%) were male, and the mean age was 63.3 years. Hepatitis $C$ was the leading cause of cirrhosis (73.8\%). The majority of patients (71.1\%) had cirrhosis Child-Pugh A at the time of EGD, 41 patients (6.7\%) had a history of previous upper digestive bleeding. Dyspeptic symptoms were present in five patients $(0.8 \%)$, four of them were receiving PPI at the time of EGD. Other indications to PPI therapy were a history of acid-related diseases found at previous $\operatorname{EGD~}(n=22)$ and gastrooesophageal reflux disease $(n=1)$.

Tables 1 and 2 show demographic and clinical features. EGD was performed as screening in 322 patients (52.7\%) and as a surveillance examination in 289 patients (47.3\%).

\subsection{Portal hypertension related findings}

EGD revealed the presence of EV in 413 patients (67.6\%): small varices in 232 patients (38\%), and medium/large varices in 181 (29.6\%). Red signs were present in $111 / 413$ of patients with EV (26.9\%), and were correlated with variceal size: $94 \%$ versus $6 \%$ $(p<0.001)$ respectively in patients with medium/large EV and small varices. In the group of patients undergoing EGD for screening $(n=322), 183$ subjects (57\%) had EV, and medium/large EV were identified in 57 patients (18\%), red signs were described in 35/183 of patients with EV (19\%).

Gastric varices were observed in 41 patients (6.7\%); 13/322 subjects (4\%) were in the screening group.

PHG was reported in 448 patients (73.3\%), with the similar rates for mild (37.3\%) and severe PHG (36\%). Among the 322 patients who underwent EGD for screening, 217 (67\%) showed evidence of PHG, mild in $36 \%$ and severe in $31 \%$ of the cases. 
Table 1

Demographic features of the 611 patients.

\begin{tabular}{ll}
\hline Parameter & \\
\hline Age (years) & $63.3 \pm 10.3$ \\
Male/female & $346 / 265(56.6 \% / 43.4 \%)$ \\
Aetiology of liver disease & \\
Hepatitis C & $451(73.8 \%)$ \\
Alcohol & $53(8.7 \%)$ \\
Hepatitis B & $50(8.2 \%)$ \\
AlH/PBC/PSC & $16(2.6 \%)$ \\
Cryptogenic & $13(2.1 \%)$ \\
Post-NAFLD & $27(4.4 \%)$ \\
Wilson's disease & $1(0.1 \%)$ \\
Child-Pugh class & \\
A & $435(71.1 \%)$ \\
B & $140(23 \%)$ \\
C & $36(5.9 \%)$ \\
\hline
\end{tabular}

Data presented as mean \pm standard deviation or $N(\%)$.

a $\mathrm{AIH}=$ autoimmune hepatitis; $\mathrm{PBC}=$ primary biliary cirrhosis; $\mathrm{PSC}=$ primary sclerosing cholangitis.

b Non-alcoholic fatty liver disease.

Table 2

Clinical features of the 611 patients.

\begin{tabular}{|c|c|}
\hline \multicolumn{2}{|l|}{ Parameter } \\
\hline Haemoglobin (g/dL) & $12.6 \pm 2.2$ \\
\hline Platelets $\left(\times 10^{3} / \mathrm{mL}\right)$ & $107 \pm 66$ \\
\hline Bilirubin (mg/dL) & $1.6 \pm 2.1$ \\
\hline $\mathrm{INR}^{\mathrm{a}}$ & $1.1 \pm 0.2$ \\
\hline Albumin (g/dL) & $3.7 \pm 0.6$ \\
\hline Spleen diameter $(\mathrm{cm})$ & $14.7 \pm 3.5$ \\
\hline $\mathrm{PPI}^{\mathrm{b}}$ at EGD & $113(18.5 \%)$ \\
\hline $\mathrm{NSAIDs}^{\mathrm{c}}$ at EGD & $9(1.5 \%)$ \\
\hline Anticoagulants at EGD & $5(0.8 \%)$ \\
\hline Beta-blocker at EGD (\%) & $56(9.2 \%)$ \\
\hline
\end{tabular}

The prevalence rates of EV, gastric varices and PHG were significantly higher in patients in Child-Pugh classes $B$ and $C$ than in patients in class A; moreover, as expected, significative associations were found among EV size, gastric varices, severity of PHG and impaired liver function tests, anaemia, low platelet count and enlarged spleen diameter (data not shown).

\subsection{Endoscopic findings unrelated to portal hypertension (Table 3)}

Overall, 232 patients (38\%) presented with endoscopic lesions not related to portal hypertension: peptic diseases $(n=193)$, proliferative diseases $(n=27)$ and vascular diseases $(n=12)$. In the screening group, $127 / 322$ patients (39.4\%) had pathologic lesions: among the 322 patients, $42 / 75$ subjects (56\%) presented with upper digestive lesions, without endoscopic findings of portal hypertension.

Acid-related conditions: Oesophagitis was reported in 32 patients (5.2\%). Gastritis was reported in 138 patients (22.6\%), 58 of whom presented with single or multiple erosions. Gastric inflammation of any degree was histologically diagnosed in all 43 cases in which biopsies were performed. Complete intestinal metaplasia was found in 3 patients, 1 of whom underwent EGD for screening. 39 patients $(6.4 \%)$ received a diagnosis of duodenitis, confirmed by histology in all 8 cases in which biopsies were performed; in 14 patients single or multiple erosions were found. In 23 patients (3.8\%), EGD revealed a gastric ulcer, multiple ulcers were found in 11 patients. A duodenal ulcer was reported in 11 patients (1.8\%).
Table 3

Endoscopic findings not related to portal hypertension.

\begin{tabular}{lll}
\hline & All subjects $(n=611)$ & Screening group $(n=322)$ \\
& $N(\%)$ & $N(\%)$ \\
\hline Oesophagitis & $32(5.2 \%)$ & $19(6 \%)$ \\
Grade A & 17 & 10 \\
Grade B & 13 & 8 \\
Grade D & 2 & 1 \\
Gastric ulcer & $23(3.8 \%)$ & $16(5 \%)$ \\
Forrest IIb & 1 & 1 \\
Forrest IIc & 5 & 4 \\
Forrest III & 17 & 11 \\
Gastritis & $138(22.6 \%)$ & $84(26 \%)$ \\
Gastric polyp & $20(3.3 \%)$ & $9(2.8 \%)$ \\
Gastric cancer & $2(0.3 \%)$ & $1(0.3 \%)$ \\
GAVE & $11(1.8 \%)$ & $3(0.9 \%)$ \\
Duodenal ulcer & $11(1.8 \%)$ & $5(1.5 \%)$ \\
Forrest IIb & 2 & 2 \\
Forrest IIc & 2 & 1 \\
Forrest III & 7 & 2 \\
Duodenitis & $39(6.4 \%)$ & $23(7.1 \%)$ \\
Duodenal polyp & $5(0.8 \%)$ & $3(0.9 \%)$ \\
Duodenal angiodisplasia & $1(0.2 \%)$ & - \\
Total number of lesions & 282 & 163 \\
Total number of patients & $232 / 611(38 \%)$ & $127 / 322(39.4 \%)$ \\
\hline a Gastric antrat vascular &
\end{tabular}

a Gastric antral vascular ectasia.

H. pylori was checked for in 68 patients with peptic ulcer, gastritis and/or duodenitis: infection was diagnosed in 51 patients (75\%), and in $37 / 44$ patients (84\%) in the screening group. H. pylori was detected in $47 \%$ of 34 peptic ulcers, and in $48 \%$ and $45 \%$ of gastric and duodenal ulcers, respectively. No significant associations were found among $H$. pylori infection, sex, age, Child-Pugh class and endoscopic signs of portal hypertension. Only 9 patients (4.7\%) with peptic diseases had alcoholic cirrhosis $(p=0.01)$; no association were found neither with other aetiologies, nor with NSAIDs or PPI intake. Screening for EV was the most frequent indication for EGD, involving $61 \%$ of the patients with peptic diseases.

Acid-related conditions were significantly correlated with compensated liver disease, no endoscopic signs of portal hypertension, higher platelet count and lower spleen diameter (Table 4). On multivariate analysis, only the absence of PHG (RR 3.3; 95\% CI 2.2-4.8) was independently associated with peptic diseases, while gastric varices, Child-Pugh class and spleen diameter had no impact on peptic diseases.

Proliferative diseases: A gastric cancer (histology: adenocarcinoma) was found in two patients (0.3\%). One of them underwent EGD for screening. Gastric polyps were reported in 20 patients (3.3\%), with a mean size of $9 \mathrm{~mm}$. Histology revealed 7 hyperplastic polyps, 3 xanthomas and 1 serrated adenoma, the last 1 found in a patient in the screening group. Multiple polyps were found in 7 patients. 5 patients presented duodenal polyps ( $0.8 \%)$, with a mean size of $11 \mathrm{~mm}$. In one of these patients who underwent EGD for screening, a polypoid lesion of the major ampulla was found, and a histological diagnosis of ampullary adenoma was made. On univariate analysis no significant associations were found with liver disease characteristics, biochemical parameters and signs of portal hypertension.

Vascular alterations: 11 patients (1.8\%) had GAVE at EGD confirmed by histology, three of them were in the screening group; one patient presented with duodenal angiodysplasia $(0.2 \%)$. Vascular lesions were seemingly correlated with EV and severe PHG (Table 5). When multivariate analysis was carried out no significant associations were found.

Operative EGDs were performed in 96/232 patients (41.4\%) with lesions not related to portal hypertension: biopsies were performed in 81 patients, polipectomy in 12 patients, injective haemostasis in 3 patients with ulcer lesions and adherent clots. Overall, a medical 
Table 4

Univariate analysis of factors associated with peptic diseases.

\begin{tabular}{|c|c|c|c|}
\hline Variable & Peptic disease present $(n=193)$ & No peptic diseases $(n=418)$ & $p$ value \\
\hline Oesophageal varices & $111(57.5 \%)$ & $302(72.2 \%)$ & $<0.001$ \\
\hline Gastric varices & $6(3.1 \%)$ & $35(8.4 \%)$ & 0.01 \\
\hline $\mathrm{PHG}^{\mathrm{a}}$ & $109(56.5 \%)$ & $339(81.1 \%)$ & $<0.001$ \\
\hline Class A Child-Pugh & $152(78.7 \%)$ & $283(67.7 \%)$ & 0.005 \\
\hline Haemoglobin (g/dL) & $12.8 \pm 2.2$ & $12.5 \pm 2.2$ & 0.07 \\
\hline Platelets $\left(\times 10^{3} / \mathrm{mL}\right)$ & $115 \pm 83$ & $103 \pm 56$ & 0.029 \\
\hline Bilirubin (mg/dL) & $1.5 \pm 1.7$ & $1.7 \pm 2.3$ & 0.289 \\
\hline $\mathrm{INR}^{\mathrm{b}}$ & $1.1 \pm 0.2$ & $1.1 \pm 0.1$ & 0.499 \\
\hline Albumin (g/dL) & $3.8 \pm 0.6$ & $3.6 \pm 0.6$ & 0.003 \\
\hline Spleen diameter $(\mathrm{cm})$ & $14.2 \pm 3.2$ & $14.8 \pm 3.6$ & 0.039 \\
\hline
\end{tabular}

Data presented as mean \pm standard deviation or $N(\%)$.

a Portal hypertensive gastropathy.

b International normalized ratio.

Table 5

Univariate analysis of factors associated with vascular diseases.

\begin{tabular}{|c|c|c|c|}
\hline Variable & Vascular diseases present $(n=12)$ & No vascular diseases $(n=599)$ & $p$ value \\
\hline Oesophageal varices & $12(100 \%)$ & $401(67 \%)$ & 0.01 \\
\hline Gastric varices & $2(16.7 \%)$ & $39(6.5 \%)$ & 0.16 \\
\hline Severe $\mathrm{PHG}^{\mathrm{a}}$ & $9(75 \%)$ & $211(35.2 \%)$ & 0.01 \\
\hline Class A Child-Pugh & $8(67.7 \%)$ & $427(71.3 \%)$ & 0.72 \\
\hline Haemoglobin (g/dL) & $11.7 \pm 2.6$ & $12.6 \pm 2.2$ & 0.13 \\
\hline Platelets $\left(\times 10^{3} / \mathrm{mL}\right)$ & $115 \pm 52$ & $107 \pm 66$ & 0.68 \\
\hline Bilirubin (mg/dL) & $1.5 \pm 1.1$ & $1.6 \pm 2.1$ & 0.84 \\
\hline $\mathrm{INR}^{\mathrm{b}}$ & $1.1 \pm 0.2$ & $1.1 \pm 0.2$ & 0.95 \\
\hline Albumin (g/dL) & $3.3 \pm 0.6$ & $3.7 \pm 0.6$ & 0.07 \\
\hline Spleen diameter $(\mathrm{cm})$ & $15.2 \pm 2.7$ & $14.7 \pm 3.6$ & 0.61 \\
\hline
\end{tabular}

Data presented as mean \pm standard deviation or $N(\%)$.

a Portal hypertensive gastropathy.

b International normalized ratio.

treatment with PPI was started in $159 / 232$ patients (68.5\%) and 1 patient with gastric cancer underwent to surgery.

\subsection{Development of new lesions in the course of endoscopic surveillance}

300 patients (49.1\%) had no upper digestive lesions unrelated to portal hypertension at the first endoscopic examination. 55 of them underwent a second EGD during the follow-up (mean $13.3 \pm 11.24$ months). Nine patients (16.3\%) developed new lesions: one oesophagitis, six gastritis, one gastric polyp (histology: hyperplastic) and one duodenitis. In all these subjects EGD was performed for endoscopic surveillance or treatment of EV.

\section{Discussion}

The prevalence rates of oesophagogastric varices and PHG in our study were comparable to those reported in the literature $[16,26,31]$. Likewise, the association between endoscopic findings of portal hypertension and severity of liver disease confirms previous data $[6,16]$. An interesting finding was the significant correlation between presence of PHG and anaemia, not found in a previous study [17], probably because of the higher prevalence of severe PHG in our study than in the HALT-C cohort.

Endoscopic findings unrelated to portal hypertension were reported in $38 \%$ of all patients with cirrhosis, and in $39.4 \%$ of those undergoing EGDs for the screening of portal hypertension. It is important to note that the majority of patients did not have at the time of endoscopy, symptoms specifically suggestive of upper digestive lesions and the $76 \%$ of patients receiving PPI did not present acid-related diseases at the time of EGD. The overall prevalence of peptic diseases was $31.6 \%$ and, in contrast to previous reports [32,33], there was no significant association with alcoholic cirrhosis or with NSAIDs intake, probably because of the small num- ber of patients with active alcohol consumption and of patients receiving these drugs at the time of EGD. Among patients with peptic diseases, the most frequent indication for EGD was screening for portal hypertension.

Even if the way to diagnose gastroduodenitis could be questionable, it is worth to note that this is a descriptive endoscopic study aimed to identify lesions requiring a therapy in a clinical setting where is essential to weigh the risk associated to a biopsy versus the benefit of a definite pathologic diagnosis.

Univariate analysis suggested that peptic diseases were significantly associated with compensated cirrhosis and no endoscopic signs of portal hypertension. Moreover, multivariate modelling confirmed that patients without PHG had a 3.3-fold higher rate of peptic diseases.

We speculated about a possible selection bias to explain these findings, but patients were specifically asked about gastrointestinal disorders at the time of EGD, and only 3/101 patients with no endoscopic findings of portal hypertension at a previous EGD referred symptoms.

These results confirm earlier studies concerning the lack of association among peptic diseases, the severity of liver disease and HVPG values [32,34]. However, other studies have reported the existence of such relationships $[35,36]$ and the pathogenesis of peptic lesions in cirrhosis is still controversial.

In our cohort the prevalence of peptic ulcers was $5.6 \%$ consistent with earlier reports $[17,37]$ and higher than in normal volunteer populations [38]. Interestingly, we found a higher prevalence of gastric ulcers than duodenal ones, differently from the majority of the studies in this setting $[17,32,35,37]$, but consistent with the results of an Italian series of 226 cirrhotic patients [39].

Peptic ulcer account for $16 \%$ of overall upper digestive haemorrhages in cirrhotics [40] and assuming a 5-fold increase in risk of complications or death for patients with cirrhosis and bleeding peptic ulcer [41], early diagnosis and antisecretory 
treatment of asymptomatic peptic ulcers could have major relevance.

Several studies have reported either increased or similar prevalence of $H$. pylori in cirrhotics when compared with controls $[35,39,42,43]$. In our study, overall prevalence of $H$. pylori infection was $75 \%$ consistent with literature data, but only $47 \%$ of peptic ulcers were related to $H$. pylori, such relatively low prevalence can be partially explained by the different methods used for the diagnosis of $H$. pylori infection, indeed, serologic studies reported higher prevalence than studies that used invasive methods [39,42-45]. Another reason could be that only $0.8 \%$ of our patients presented dyspeptic symptoms at the time of EGD. Moreover, history of antibiotics intake could affect the prevalence of $H$. pylori, but, due to its retrospective nature, the present study cannot assess this variable. Triple therapy for $H$. pylori in cirrhotic patients achieves high rates of eradication and prevents ulcer recurrence $[46,47]$. Since $H$. pylori is involved in gastric carcinogenesis, and the prevalence of gastric cancer in cirrhotics is higher than in the general population, its eradication could prevent development of gastric adenocarcinoma $[19,48]$. Of the two gastric cancer cases $(0.3 \%)$ found in this cohort, one was seen as an incidental lesion in a patient underwent EGD for screening. We found an overall prevalence of gastric polyps of $3.3 \%$ in our patients, higher than in previously reported series in cirrhotics, which ranged from 1.3 to $2 \%[37,49]$ and in the general population [50]. Adenomatous polyps are premalignant lesions, and after excision an endoscopic surveillance program is recommended to exclude recurrence [51]. Hyperplastic polyps may have increased risk of cancer, and harbour dysplastic foci in up to $19 \%$ of cases. Some authors recommend polypectomy of all lesions of $>0.5 \mathrm{~cm}$ [52]. In our study, histology revealed one incidental adenomatous polyp (5\%) in one patient undergoing EGD for screening, and hyperplastic polyps in $35 \%$ of the cases. Other premalignant conditions, such as intestinal metaplasia associated with $H$. pylori $(n=3)$, and ampullary adenoma $(n=1)$, were diagnosed incidentally; two of the four patients underwent EGD for screening.

GAVE was the most frequent vascular disease, reported in $1.8 \%$ of patients. A significant correlation was found among vascular diseases, presence of EV and severe PHG. However multivariate analysis did not confirmed these findings. There was no significant difference in haemoglobin levels according to the presence of vascular diseases, probably because the majority of the lesions were not actively bleeding at the time of EGD. This result suggests that in cirrhotic patients vascular lesions can be present regardless of severe anaemia.

During surveillance, $16 \%$ of patients in the subgroup without previous lesions unrelated to portal hypertension, developed new lesions but considering the small size of this subgroup such result cannot support definite conclusions about incidence of new lesions.

This study involved more than 600 consecutive patients and it inspects the wide range of endoscopic findings in cirrhotics; however the sample size of some subgroups might be too small to allow robust conclusions.

A further limitation is represented by the retrospective design with no control population, though this study reflects the real clinical practice in a single, tertiary referral centre for liver cirrhosis. Finally factors as aetiology and severity of liver cirrhosis, diagnostic methods, geographic and racial differences may explain discrepancies in our results.

The methods proposed as alternatives to EGD for the screening of patients with cirrhosis and portal hypertension, based on ultrasound, biochemistry or transient elastometry, are in principle unsuitable for detecting, in this setting, pathologic findings unrelated to portal hypertension. MCT oesophagography cannot reveal EV bleeding stigmata, found in 19\% of the patients in our screening group, vascular diseases and small sized proliferative or peptic lesions. Capsule endoscopy is the only technique capable of detecting EV red signs and other endoscopic lesions, though like CT scanning it lacks therapeutic potential.

Although only a minority of patients in the screening group had EV requiring prophylactic therapy (18\%), in $39.4 \%$ of patients in this group, EGD resulted in changes in management, consisting in a new therapy, polypectomy or surgery for digestive cancer. In our opinion, EGD still plays a major role in a careful evaluation of the patient with cirrhosis, and offers the advantage over other diagnostic tools of allowing clinicians to perform biopsies and carry out therapeutic interventions in a timely manner.

\section{Conflict of interest}

The authors declare that they have no conflict of interest.

\section{Acknowledgement}

The authors declare that they did not receive funding in this study. The authors would like to thank Piero Luigi Almasio for his statistical advices and Warren Blumberg for his help in editing this paper.

\section{References}

[1] Jalan R, Hayes PC. UK guidelines on the management of variceal haemorrhage in cirrhotic patients. Gut 2000;46:S1-15.

[2] de Franchis R. Updating consensus in portal hypertension. J Hepatol 2000;33:846-52.

[3] de Franchis R. Evolving consensus in portal hypertension. Report of the Baveno IV Consensus Workshop on methodology of diagnosis and therapy in portal hypertension. J Hepatol 2005;43:167-76.

[4] Garcia-Tsao G, Sanyal AJ, Grace ND, et al. Prevention and management of gastrooesophageal varices and variceal hemorrhage in cirrhosis. Hepatology 2007;46:922-38.

[5] Qureshi W, Adler DG, Davila R, et al. ASGE guideline: the role of endoscopy in the management of variceal hemorrhage, updated July 2005. Standards of Practice Committee. Gastrointest Endosc 2005;62:651-5.

[6] Pagliaro L, D’Amico G, Pasta L, et al. Portal hypertension in cirrhosis: natural history. In: Bosch J, Grozmann RJ, editors. Portal hypertension. Pathophysiology and treatment. Oxford, UK: Blackwell Scientific; 1994. p. 72-92.

[7] Giannini E, Botta F, Borro P, et al. Platelet count/spleen diameter ratio: proposal and validation of a non-invasive parameter to predict the presence of oesophageal varices in patients with liver cirrhosis. Gut 2003;52:1200-5.

[8] Thabut D, Trabut JB, Massard J, et al. Non-invasive diagnosis of large oesophageal varices by fibrotest in patients with cirrhosis: a preliminary retrospective study. Liver Int 2006;26:271-8.

[9] Vizzutti F, Arena U, Romanelli RG, et al. Liver stiffness measurement predicts severe portal hypertension in patients with HCV-related cirrhosis. Hepatology 2007;45:1290-7.

[10] Bureau C, Metivier S, Peron JM, et al. Transient elastography accurately predicts presence of clinically significant portal hypertension in patient with chronic liver disease. Aliment Pharmacol Ther 2008;27:1261-8.

[11] Kim SH, Kim YJ, Lee JM, et al. Oesophageal varices in patients with cirrhosis: multidetector CT esophagography-comparison with endoscopy. Radiology 2007;242:759-68.

[12] Perri RE, Chiorean MV, Fidler JL, et al. A prospective evaluation of computerized tomographic scanning as a screening modality for oesophageal varices. Hepatology 2008;47:1587-94.

[13] de Franchis R, Eisen GM, Laine L, et al. Oesophageal capsule endoscopy for screening and surveillance of oesophageal varices in patients with portal hypertension. Hepatology 2008;47:1595-603.

[14] Spiegel BMR, Esrailian E, Eisen G. The budget impact of endoscopic screening for oesophageal varices. Gastrointest Endosc 2007;66:679-769.

[15] D'Amico G, Montalbano L, Traina M, et al. Natural history of congestive gastropathy in cirrhosis. Gastroenterology 1990;99:1558-64.

[16] Primignani M, Carpinelli L, Preatoni P, et al. Natural history of hypertensive gastropathy in patients with liver cirrhosis. The New Italian Endoscopic Club for the study and treatment of esophageal varices. Gastroenterology 2000;119:181-7.

[17] Fontana RJ, Sanyal AJ, Mehta S, the HALT-C Trial Group. Portal hypertensive gastropathy in chronic hepatitis $C$ patients with bridging fibrosis and compensated cirrhosis: results from the HALT-C trial. Am J Gastroenterol 2006;101:983-92.

[18] Siringo S, Burroughs A, Bolondi L, et al. Peptic ulcer and its course in cirrhosis: an endoscopic and clinical prospective study. J Hepatol 1995;22:633-41.

[19] Zullo A, Romiti A, Tomao S, et al. Gastric cancer prevalence in patients with liver cirrhosis. Eur J Cancer Prev 2003;12:179-82.

[20] Hennes EM, Zeniya M, Czaja AJ, et al. Simplified criteria for the diagnosis of autoimmune hepatitis. Hepatology 2008;48:169-76.

[21] Lindor KD, Gershwin ME, Poupon R, et al. American Association for Study of Liver Diseases. Primary biliary cirrhosis. Hepatology 2009;50:291-308. 
[22] Silveira MG, Lindor KD. Primary sclerosing cholangitis. Can J Gastroenterol 2008;22:689-98.

[23] National Cholesterol Education Program (NCEP) Expert Panel on detection, evaluation, and treatment of high blood cholesterol in adults (Adult Treatment Panel III). Third report of the National Cholesterol Education (NCEP) Expert Panel on detection, evaluation, and treatment of high blood cholesterol in adults (Adult Treatment Panel III) final report. Circulation 2002;106:3143-421.

[24] Pugh RNN, Murray-Lyon IM, Dawson JL, et al. Transection of esophagus for bleeding esophageal varices. Br J Surg 1973;60:646-9.

[25] North Italian endoscopic club for the study and treatment of esophageal varices. Prediction of the first variceal hemorrhage in patients with cirrhosis of the liver and esophageal varices. N Engl J Med 1988;319:983-9.

[26] Sarin SK, Lahoti D, Saxena SP, et al. Prevalence, classification and natural history of gastric varices: a long term follow-up study in 568 portal hypertension patients. Hepatology 1992;16:1343-9.

[27] Carpinelli L, Primignani M, Preatoni P, et al. Portal hypertensive gastropathy: reproducibility of a classification, prevalence of elementary lesions, sensitivity and specificity in the diagnosis of cirrhosis of the liver. A NIEC multicentre study. New Italian Endoscopic Club. Ital J Gastroenterol Hepatol 1997;29:533-40.

[28] Armstrong D, Bennett JR, Blum AL, et al. The endoscopic assessment of oesophagitis: a progress report on observer agreement. Gastroenterology 1996;111:85-92.

[29] Forrest JAH, Finlayson NDC, Shearman DJV. Endoscopy in gastrointestinal bleeding. Lancet 1974;2:394-7.

[30] Jabbari M, Cherry R, Lough JO, et al. Gastric antral vascular ectasia: the watermelon stomach. Gastroenterology 1984;87:1165-70.

[31] Pique JM. Portal hypertensive gastropathy. Baillieres Clin Gastroenterol 1997;11:257-70.

[32] Rabinovitz M, Yoo YK, Schade R, et al. Prevalence of endoscopic findings in 510 consecutive individuals with cirrhosis evaluated prospectively. Dig Dis Sci 1990;35:705-10.

[33] Auroux J, Lamarque D, Roudot-Thoraval F, et al. Gastroduodenal ulcer and erosions are related to portal hypertensive gastropathy and recent alcohol intake in cirrhotic patients. Dig Dis Sci 2003;48:1118-23.

[34] McCormack TT, Sims J, Eyre-Brook I, et al. Gastric lesions in portal hypertension: inflammatory gastritis or congestive gastropathy? Gut 1985;26:1226-32.

[35] Wu CS, Lin CY, Liaw YF. Helicobacter pylori in cirrhotic patients with peptic ulcer disease: a prospective, case controlled study. Gastrointest Endosc 1995;42:424-7.

[36] Chen LS, Lin HC, Hwang SJ, et al. Prevalence of gastric ulcer in cirrhotic patients and its relation to portal hypertension. J Gastroenterol Hepatol 1996;11:59-64.
[37] Zaman A, Hapke R, Flora K, et al. Prevalence of upper and lower gastrointestinal tract findings in liver transplant candidates undergoing screening endoscopic evaluation. Am J Gastroenterol 1999;94:895-9.

[38] Akdamar K, Ertan A, Agrawal NM, et al. Upper gastrointestinal endoscopy in normal asymptomatic volunteers. Gastrointest Endosc 1986;32:78-80.

[39] Zullo A, Rinaldi V, Meddi P, et al. Helicobacter pylori infection in dyspeptic cirrhotic patients. Hepato-Gastroenterology 1999;46:395-400.

[40] Dagradi AE, Mehler R, Tan ATD, et al. Sources of upper gastrointestinal bleeding in patients with liver cirrhosis and large esophageal varices. Am J Gastroenterol 1970;54:458-63.

[41] Bonnevie O. Causes of death in duodenal and gastric ulcer. Gastroenterology 1977;73:1000-4.

[42] Siringo S, Vaira D, Menegatti M, et al. High prevalence of Helicobacter pylori in liver cirrhosis. Relationship with clinical and endoscopic features and the risk of peptic ulcer. Dig Dis Sci 1997;42:2024-30.

[43] Pellicano R, Leone N, Berrutti M, et al. Helicobacter pylori seroprevalence in hepatitis C virus positive patients with cirrhosis. J Hepatol 2000;33:648-50.

[44] Wang CH, Ma LR, Lin RC, et al. Helicobacter pylori infection and risk of peptic ulcer among cirrhotic patients. J Formos Med Assoc 1997;96:55-8.

[45] Shahin WA, Abdel Baset EZ, Nassr AK, et al. Low incidence of Helicobacter pylori infection in patients with duodenal ulcer and chronic liver disease. Scand J Gastroenterol 2001;36:479-84.

[46] Miquel J, Barcena R, Boixeda D, et al. Role of Helicobacter pylori infection and its eradication in patients with subclinical hepatic encephalopathy. Eur J Gastroenterol Hepatol 2001;13:1067-72.

[47] Damirturk L, Yazgan Y, Izci O, et al. The effect of Helicobacter pylori eradication on gastric juice and blood ammonia concentrations and on visual evoked potentials in cirrhotics. Helicobacter 2001;6:325-30.

[48] Sorensen HT, Friis S, Olsen JH, et al. Risk of liver and other types of cancer in patients with cirrhosis: a nationwide cohort study in Denmark. Hepatology 1998;28:921-5.

[49] Svoboda P, Ehrmann J, Klvana P, et al. Endoscopic findings in upper gastrointestinal tract in patients with liver cirrhosis. Vnitr Lek 2007;53:968-71.

[50] Morais DJ, Yamanaka A, Zeitune JM, et al. Gastric polyps: a retrospective analysis of 26,000 digestive endoscopies. Arq Gastroenterol 2007;44:14-7.

[51] Hirota WK, et al. ASGE guideline: the role of endoscopy in the surveillance of premalignant conditions of the upper GI tract. Gastrointest Endosc 2006;63:570-80.

[52] Ginsberg GG, Al-Kawas FH, Fleischer DE, et al. Gastric polyps: relationship of size and histology to cancer risk. Am J Gastroenterol 1996;91:714-7. 